variation of pressure at Aberdeen over each of two years of low sunspot number, 1934 (of mean magnetic character figure $0 \cdot 56$ ), and 1942 (mean character figure $0 \cdot 65$ ). Here the curve for the year of higher mean magnetic activity shows a marked enhancement of the forenoon maximum and reduced development of the evening maximum as compared with the year of low magnetic activity - a result opposite to that found by Stagg for selected quiet and disturbed days. Other similar examples are to be found in the twentytwo years examined, and also still others in which the changes of type of diurnal pressure variation with magnetic activity are in the opposite sense to that of Hig. 2. 'The truth appears to be that a sample of even 365 days is too small to remove, from the form of the diurnal pressure variations, features that must be considered purely random from the point of view of magnetic activity. This result agrees with that obtained from the independent test described in the preceding paragraph.

R. P. Waldo Lewis

D. H. MeIntosh

Meteorological Office, Edinburgh Jan. 16.

1 Stagg, J. M., Nature, 127, 402 (1931).

${ }^{2}$ Chapman, S., and Bartels, J., "Geomaguetism", 1, 204 (1940).

\section{Measurements of Amplitudes of Micro- Oscillations using a Microscope Light-Cut Technique}

Ir has recently been shown by Tolansky ${ }^{1}$ how the Schmaltz light-cut technique can be modified and developed such that magnification of $\times 2,000$ with resolutions of $0.25 \mu$ is obtainable. We have applied one of these modified methods to the measurement of the amplitudes of vibration of a $20-\mathrm{cm}$. Monel rod excited by the familiar magneto-striction methods. Tolansky and Bardsley ${ }^{2}$ have already shown how the small amplitudes of vibration usually met with in quartz vibrators (ranges of the order of 50-5000 A.) can be measured interferometrically, but this is a relatively elaborate technique. 'I'he amplitudes which can safely be developed in a magneto-striction vibrator can well exceed several microns, and for measurements of such amplitudes, interferometry is neither necessary nor desirable in view of the simplicity of the high-resolution light-cut method already reported on by one of us.

'i'he accompanying illustrations indicate the value of the method. The end of the vibrating rod is
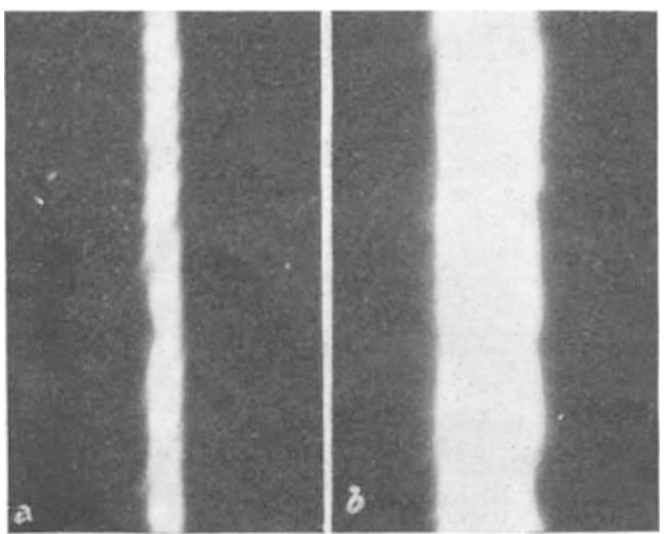

polished reasonably smooth (or can have cemented on to it a piece of a cover slip). When viewed with light-cut illumination with a broad slit and a 4-mm. microscope objective, the straight line image $(a)$ is observed. The roughness of polish can be seen. On setting the rod into vibration, the light-cut profile becomes the widened rectangular pattern shown at (b). With the particular lens systems in use for this profile, magnification in depth is $\times 350$. Hence from the width of $(b)$ (which on the original plate is $2.8 \mathrm{~mm}$.) it follows that the amplitude of vibration normal to the surface in this specific instance is 10 microns. (The central region is of lower intensity, but this is not easily visible on the photograph.)

By traversing the specimens across the field of view, the distribution of oscillation is measurable. If damping is undesirable, then oil immersion is avoided, which restricts useful magnification to the order of $\times 1,000$. Even so, this magnification in extension is greater than the magnification in extension across the surface that can usefully be employed with multiple-beam interferometry. The light-cut can give useful magnification both in depth and in extension simultaneously of some $\times 2,000$ and has the merit of simplicity. Hence for vibrations of amplitude exceeding one micron it offers decided advantages.

S. 'Tolansky

Royal Holloway College

(University of London),

Englefield Green, Surrey. March 10.

${ }^{1}$ Tolansky, S., Nature [169, 455 (19j2)]; payer before Physical Society on September 20,1951 . 2 Proc. Phys. Soc., 62B, 224 (1951).

\section{Light Emission from Polonium}

THE visible spectrum of light emitted from a sample of polonium has been photographed using the night-sky spectrograph designed by McLennan and Ireton ${ }^{1}$. The activity of the sample was about $4 \mathrm{mC}$., and it was deposited on a platinum sheet, $3 \mathrm{~mm}$. $\times$ $3 \mathrm{~mm}$. This shape was chosèn for another purpose and is not very suitable for a spectral investigation. The night-sky spectrograph contains a glass prism and, therefore, its application is restricted to the visible range. The aperture is $f / 1$. The slit-width used in this investigation was $0.4 \mathrm{~mm}$. The polonium sample was put in two positions with respect to the slit of the spectroscope : (1) the surface of the sample was in the plane of the slit parallel to the collimator axis so that light emitted from the surface could enter the slit ; (2) the surface was about $1 \mathrm{~mm}$. below the slit, so that only light emitted from the volume of ionized gas (air) above the surface could enter the slit.

The exposure time was always about one week in a room carefully darkened. An exposure without the sample gave no effect on the photographic plate. 'i'he plate type used was Gevaert Panchromosa.

In position (1), some lines, mostly weak, were detected on the plate, superposed on a background of continuous bands showing a varying intensity. The wave-lengths of the lines are collected in Table 1, with also an estimate of the intensities and the probable interpretation according to $H$. Kayser's tables ${ }^{2}$. 